LIST OF NEW ANTIBIOTICS AND THEIR DERIVATIVES DISCOVERED

BY DR. HAMAO UMEZAWA

Antitumor Antibiotics:

No. 289 Substance (1953), Sarkomycin (1953), Actinoleukin (1954), Ractinomycins A and B (1955), Pluramycins A and B (1956), Phleomycin (1957), Raromycin (1957), No. 418 Substance (1959), Peptimycin (1961), Enomycin (1963), Labilomycin (1963), Hilamycin (1963), Formycin A (1964), Bleomycins (1965), Plurallin (1966), Phenomycin (1967), Macromomycin (1968), Acrylamidine (1968), Coriolins (1969), Neopluramycin (1970), Diketocoriolin B (1971), Macracidomycin (1975), Glyoxalase Inhibitor (1975), Aclacinonycins A and B (1975), Glyoxalase Inhibitor MS-3 (1975), Neothramycin (1976), Rhodirubins (1977), Baumycins (1977).

\title{
Antimicrobial Antibiotics:
}

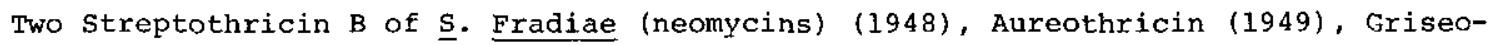
lutein A and B (1950), Nitrosporin (1951), Actinomycin J (1951), Abikoviromycin (1952), Exfoliatin (1952), Moldin (1952), Phaeofacin (1952), Sarcidin (1953), Achromoviromycin (1953), Thiazolidone Antibiotic (1953), Pyridomycin (1953), Azomycin (1953), Phthiomycin (1953), Seligocidin (1954), Aureothin (1954), Mediocidin (1954), Tertiomycin A and B (1955), Antitoxoplasmic Substance No. 534 (1955), Mesenterin (1955), Kanamycin A, B and C (1957), Althiomycin (1957), Alboverticillin (1958), Mikamycin (1959), Blastmycin (1957), Nixomycin A and B (1960), Unamycin A and B (1960), Amidinomycin (1960), Emimycin (1960), Ilamycin A1, A2, B1, B2, C1 and C2 (1961), Cytomycin (1961), Griseococcin (1962), Monazomycin (1963), Bottromycin Al, A2 and B (1965), Kasugamycin (1965), Spinanycin (1966), Josamycin (1967), Leucinamycin (1967), Pepthiomycin (1968), Ablastmycin (1968), Laspartomycin (1968), Oryzoxymycin (1968), Gougeroxymycin (1969), Deoxynybomycin (1970), Macarbomycin (1974), Oryzoxymycin (1974), Amiclenomycin (1974), Calvatic Acid (1975), SS-228Y (1975), Aplasmomycin (1977), B-Lactamase inhibitors (1977), 3-epi-Deoxynegamycin (1977), Leucy 1-3-epinegamycin (1977), Pheganomycin (1978), Aplasmomycins B and C (1978).

Derivatives of Aminoglycosidic Antibiotics: 3',4'-Dideoxykanamycin B (1.971), 5"-Deoxylividomycin A (1972), 5"-Amino-5"-deoxylividomycin A (1972), 5"-Deoxylividomycin B (1972), 3',4'-Dideoxy-6-N-methylkanamycin B (1972), 3'-Deoxykanamycin (1972), 3'-O-Methylkanamycin (1972), 1-N-(4- 
Amino-2-hydroxybutyryl) lividomycin A (1973), 3',4'-Dideoxybutirosin B (1973), 1-N(4-Amino-2-hydroxybutyry1)-kanamycin B (1973), 3'-Deoxybutirosin B (1973), 1-N-(4Amino-2-hydroxybutyryl)-6-N-methy 1-3', $4^{\prime}$-dideoxykanamycin B (1973), 6'-Amino-6 deoxyluvidomycin B (1973), 6'-Deoxy-6'-methylaminolividomycin B (1973), 6'-Deoxy6'-hydroxyethylaminolividomycin B (1973), l-N-Isoserylkanamycins (1974), 4'-Deoxykanamycin (1974), 1-N-[(S)-4-amino-2-hydroxybutyryl]-3',4'-dideoxyneamine (1974), 1-N-acyl-3', 4'-dideoxy-6'-N-methylkanamycin B (1975), 1-N- [ (S)-4-amino-2-hydroxybutyryl]-6'-N-alkylkanamycin (1975), 3'-deoxybutirosin B (1975), 3"-deoxydihydrostreptomycin (1976), lividomycin derivatives (1976).

Enzyme Inhibitors:

Leupeptin (1969) inhibiting trypsin, plasmin, papain, thrombokinase and cathepsin $B$, Antipain (1972) inhibiting trypsin, papain, thrombokinase, cathepsin $A$ and cathepsin B, Chymostatin (1970) inhibiting chymotrypsins, papain and cathepsin B, Elastatinal (1973) inhibiting elastase, Pepstatin (1970) inhibiting pepsin, gastricsin, cathepsin D and renin, Hydroxy pepstatin (1973) inhibiting pepsin, gastricsin, cathepsin D and renin, Pepstanone (1972) inhibiting pepsin, gastricsin, Cathepsin D and renin, Phosphoramidon (1973) inhibiting thermolysin, Panosialin (1971) inhibiting trypsin, plasmin, pepsin, sialidase, acid phosphatase and polygalacturonase, Aquayamycin (1968) inhibiting tyrosine hydroxylase, oudenone (1970) inhibiting tyrosine hydroxylase, Fusaric acid (1969) inhibiting dopamine B-hydroxy1ase, Dopastin (1972) inhibiting dopamine B-hydroxylase, oosponol (1972) inhibiting dopamine $\beta$-hydroxylase, Pimprinine (1973) inhibiting monoamine oxidase, Cinnamic acid amide (1973) inhibiting monoamine oxidase, Methylspinazarin (1973) inhibiting catechol-o-methyltransferase, 7-0-methylspinochrome B (1973) inhibiting catechol-o-methyltransferase, Dihydromethylspinazarin (1973) inhibiting catechol-0methyltransferase, 6-(3-hydroxy-n-buty 1)-3-0-methylspinochrome B (1973) inhibiting catechol-o-methyltransferase, 1-[2-(3,4,5,6-tetrahydropyridy 1$)]-1,3-p e n t a d i e n e$ (1974) inhibiting N-methyl-transferase, Lecanoric acid (1974) inhibiting histidine decarboxylase, 5-Formyl uracil (1972) inhibiting xanthine oxidase, coformycin (1967) inhibiting adenosine deaminase, B-Lactamase inhibitor (1974), Siastatin (1974) inhibiting clostridium sialidase, Dopastin (1974) inhibiting dopamine B-hydroxylase, Pyridindolol (1975) inhibiting B-galactosidase, Reticulol (1975) inhibiting cyclic adenosine 3',5'-monophosphate phosphodiesterase, New isoflavones (1975) inhibiting catechol-0-methyltransferase, Revistin (1975) inhibiting reverse 
transcriptase, Isoflavones (1975) inhibiting dopa decarboxylase, Isoflavone rhamnosides (1975) inhibiting $\beta$-galactosidase, Bestatin (1975) inhibiting aminopeptidase $B$ and leucine aminopeptidase, Dehydrodicaffeic acid dilactone (1976) inhibiting catechol-0-methyltransferase, Tetra-o-methyldehydrodicaffeic acid dilactone (1977), 2,5-Dihydro-L-phenylalamine (1977) inhibiting tryptophan hydroxylase, Forphenicine (1978) inhibiting alkaline phosphatase of chick intestine, Esterastin (1978) inhibiting esterase, Amastatin (1978) inhibiting aminopeptidase A, B-Hydroxyphenylacetaldoxime (1979) inhibiting B-galactosidase. 